\title{
Laryngeal Stenosis, a Complication of Multifocal Tuberculosis
}

\author{
Samaké Djibril $^{1,}$, , Sidibé Youssouf ${ }^{2}$, Dienta Lassine ${ }^{3}$, Haidara Abdoul Wahab ${ }^{2}$, Kouma Alassane $^{4}$, \\ Touré Mamadou Karim ${ }^{5}$, Ouattara Kadidia ${ }^{6}$, Kanouté Tenin ${ }^{6}$, Soumaoro Siaka ${ }^{7}$, \\ Guindo Boubacary ${ }^{7}$, Traoré Lamine ${ }^{2}$, Sanogo Boubacar $^{2}$, Keita Mohamed Amadou ${ }^{7}$, \\ Ag Mohamed Alhousseini ${ }^{7}$
}

\author{
${ }^{1}$ ENT and Head and Neck Surgery Department, Commune V Reference Health Center, Bamako, Mali \\ ${ }^{2}$ ENT and Head and Neck Surgery Department, University Hospital Center Mother-Child "Le Luxembourg", Bamako, Mali \\ ${ }^{3}$ ENT and Head and Neck Surgery Department, Mopti Regional Hospital, Mopti, Mali \\ ${ }^{4}$ Medical Imaging Department, University Hospital Center Mother-Child "Le Luxembourg", Bamako, Mali \\ ${ }^{5}$ Anaesthesia and Resuscitation Department, University Hospital Center Mother-Child "Le Luxembourg", Bamako, Mali \\ ${ }^{6}$ Pneumo-phtisiology Department, University Hospital Center "Point-G”, Bamako, Mali \\ ${ }^{7}$ ENT and Head and Neck Surgery Department, University Hospital Center "Gabriel Touré”, Bamako, Mali
}

\section{Email address:}

samakedjibi@yahoo.fr (S. Djibril)

${ }^{*}$ Corresponding author

\section{To cite this article:}

Samaké Djibril, Sidibé Youssouf, Dienta Lassine, Haidara Abdoul Wahab, Kouma Alassane, Touré Mamadou Karim, Ouattara Kadidia, Kanouté Tenin, Soumaoro Siaka, Guindo Boubacary, Traoré Lamine, Sanogo Boubacar, Keita Mohamed Amadou, Ag Mohamed Alhousseini. Laryngeal Stenosis, a Complication of Multifocal Tuberculosis. International Journal of Otorhinolaryngology.

Vol. 6, No. 2, 2020, pp. 31-34. doi: 10.11648/j.ijo.20200602.13

Received: August 21, 2020; Accepted: September 3, 2020; Published: September 30, 2020

\begin{abstract}
Laryngeal stenosis is a permanent, usually acquired, narrowing of the laryngeal duct. Due to the pandemic of Acquired Immunodeficiency Syndrome (AIDS), the etiology of tuberculosis must be investigated. However, the signs of presumption are not specific. Management is controversial and remains difficult in our work context in an under-medicated country south of the Sahara. The purpose of the work is to report a case of laryngeal stenosis secondary to multifocal tuberculosis to discuss the difficulties associated with its management. This was a 35 -year-old smoking patient with 25 packs / year. Admitted on 04/12/2017 to the ENT and Head and Neck Surgery Department for assessment of dysphonia associated with unencrypted slimming. The performed laryngoscopy showed disseminated ulceration of the laryngeal margin, and the histology showed granulomatous laryngitis. The intradermal reaction to tuberculin measured $10 \mathrm{~mm}$. A chest X-ray performed on the face showed a chronic interstitial-bronchial syndrom. The diagnosis of laryngeal tuberculosis secondary to a pulmonary focus was retained. The course was marked by the sudden onset of laryngeal dyspnea requiring urgent tracheotomy. Postoperative examination of the larynx revealed synechia of the larynx. Anti-tuberculosis therapy for six months has resulted in favourable outcomes. However, it was noted a complication type laryngeal stenosis despite the attempted re-stabilization with impossibility to decant the patient. Laryngeal stenosis secondary to multifocal tuberculosis remains a rare and dreadful pathology. Therapeutic success depends on early and adequate management.
\end{abstract}

Keywords: Stenosis, Larynx, Tuberculosis

\section{Introduction}

Laryngeal stenosis is a permanent narrowing, most often acquired, of the laryngeal pathway. It is generally of progressive constitution, linked to an organic damage to the walls of the respiratory tract by a new tissue, usually fibrous, which eliminates the strictures by compression, the strictures of tumour origin and the laryngeal paralysis [1]. It is largely dominated by the sequelae of assisted ventilation [1-3]. 
Before the advent of it, most laryngeal strictures were secondary to infections such as diphtheria, measles, smallpox, typhus, syphilis, leprosy, tuberculosis. Other etiologies are rarely encountered and include certain granulomatous diseases with laryngeal expression (Wegener's disease, sarcoidosis, amyloidosis), iatrogenic causes, autoimmune diseases, postradiation stenosis, and idiopathic causes [1-4].

Laryngeal tuberculosis was the most common laryngeal disease at the start of the 20th century [5]. Its incidence then gradually decreased, in parallel with that of pulmonary tuberculosis from World War II. Due to the Acquired Immune Deficiency Syndrome (AIDS) pandemic, its incidence has been steadily increasing over the past 20 years $[6,7]$. Clinically, it manifests itself by nonspecific signs, limited to the larynx or part of a multi-organ pathology. Its eminently bacilliferous character requires rapid diagnosis and medical treatment.

Evaluating the degree and extensions of the stenosis is an essential prerequisite for any therapeutic management, the goal of which must be the restoration of a satisfactory respiratory system while preserving acceptable phonation [1]. If the etiology, pathogenesis, and clinical aspects of laryngeal stenosis are currently well known, therapeutic management remains controversial, as evidenced by the multiplicity of surgical techniques and the lack of uniformity in therapeutic management [1].

\section{Objective}

The objective of this paper was to report a case of laryngeal stenosis secondary to multifocal tuberculosis in order to discuss the difficulties associated with its management in a developing country.

\section{Observation}

This is a 35-year-old 40 packs / year smoker who has been weaned for two weeks. Admitted on 12/04/2017 to the ENT department of CHME "Luxembourg" for dysphonia. The onset of symptoms would go back to about 7 months marked by dysphonia, especially in the morning, regressing during the day. It evolved on a permanent background followed by intermittent dysphagia to solid foods often accompanied by false routes. There was no dyspnea. Uncounted weight loss, asthenia and a feeling of fever were reported.

On physical examination, the general condition was altered with a Karnoski index at $50 \%$, a weight of $48 \mathrm{~kg}$. The first laryngoscopy of 07/12/2017 showed a disseminated ulceration of the laryngeal rim and vocal folds covered with mucopurulent secretion not bleeding on contact (Figure 1).

The endo-laryngeal biopsy performed showed necrotic granulomatous laryngitis. The tuberculin intradermal reaction (IDR) measured $10 \mathrm{~mm}$. A frontal chest X-ray performed showed reticulo-micronodular infiltrates associated with thickening of the bronchial walls sparing the two pulmonary bases suggesting an infectious origin. No pleural effusion or mediastinal abnormality (Figure 2).

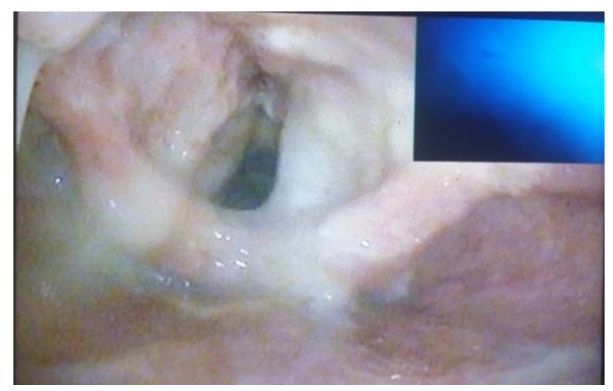

Figure 1. The laryngoscopy (Image of the larynx 07/12/2017). Disseminated ulceration of the laryngeal rim and vocal folds covered with mucopurulent secretion.

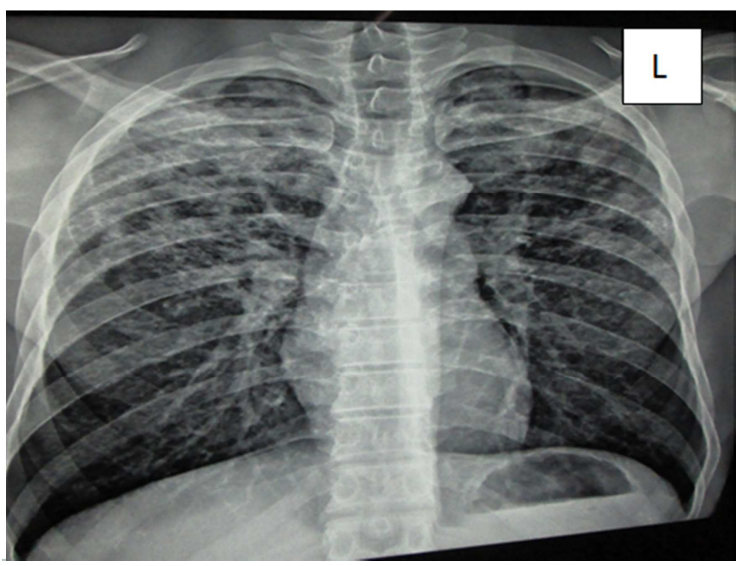

Figure 2. Chest $x$-ray Chronic interstitial bronchial syndrome predominant in the two upper lobes suggesting an infectious origin.

A gastric tube performed was positive with two crosses. The blood count revealed microcytic hypochromic anaemia and polynuclear neutrophilic hyperleukocytosis; CRP was high; HIV serology was negative and fasting blood sugar was normal.

These clinical and paraclinical elements made it possible to evoke the diagnostic hypothesis of laryngeal tuberculosis secondary to a pulmonary focus.

The patient was put on oral tuberculosis treatment for six months on the two-month regimen of Rifampicin, Isoniazid, Pyrazinamide, Ethambutol and four months of Rifampicin, Isoniazid. Two weeks after the start of this treatment, inspiratory dyspnea suddenly set in. A control laryngoscopy revealed partial synechia (posterior commissure and interarytenoid notch) of the larynx with fixation of the arytenoids, considerably reducing the laryngeal pathway (Figure 3).

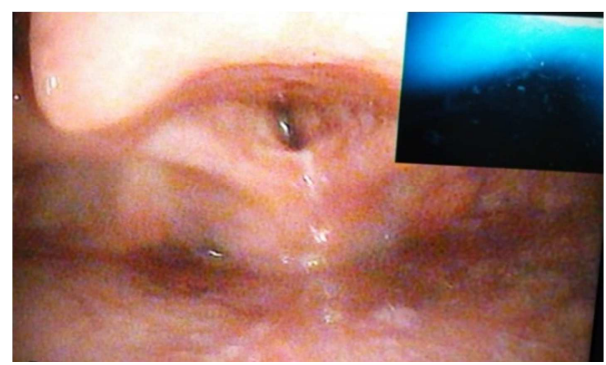

Figure 3. The control laryngoscopy: Partial synechia (posterior commissure and interarytenoid notch) of the larynx with fixation of the arytenoids, considerably reducing the glottic pathway. 
Faced with the absence of laser reconstructive microsurgery in our country, we performed symptomatic treatment such as tracheotomy.

These various treatments made it possible to obtain a favorable outcome with gradual improvement of the general condition, the disappearance of clinical signs with weight gain. However, it was noted after 12 months of evolution the persistence of certain complications such as scarring stenosis sequelae of the larynx despite attempts of permeabilization with the impossibility of decannulating the patient.

\section{Discussion}

Acquired laryngotracheal stenosis can be secondary to several etiology. However, prolonged endotracheal intubation remains the most common cause with $90 \%$ of cases [2]. In our case it was secondary to multifocal tuberculosis.

Epidemiologically, laryngeal tuberculosis affects the same populations as pulmonary tuberculosis (socially disadvantaged individuals, migrants from countries with a high tuberculosis endemic, immuno compromised individuals, health workers) [6-8]. Alcoholism and to a lesser extent smoking are two known risk factors which explain, in addition to nonspecific laryngeal clinical signs, the frequent diagnostic confusions with laryngeal squamous cell carcinoma. It is more common in men than in women (sex ratio $\mathrm{M} / \mathrm{F}$ of 2 to 16 ) [6]. The mycobacteria family includes several species including Mycobacterium tuberculosis (Koch's bacillus), agent of human tuberculosis, and Mycobacterium bovis, agent of bovine tuberculosis (exceptional in humans) [9]. The contagion is almost always human-to-human, airborne through contact with a bacilliferous subject. Only 5\% of contact individuals will develop tuberculosis disease after tuberculosis infection (due to a significant bacterial load, or during immunosuppression), either in the short term or in the long term, sometimes several decades after the initial infectious contact [6, 7]. Laryngeal tuberculosis may be the only site of infection or may occur in a multi-organ setting. In the latter case, dissemination takes place via the lymphatic or bloodstream routes and can involve all organs. However, the association of laryngeal and pulmonary involvement is the most common [10]. This infection of the cartilaginous and periarticular mucosa can lead to chondritis and periarthritis; their evolution can take place in a fibrous healing mode which can thicken the mucosa or create cricoarytenoid ankylosis, constitutive of laryngeal stenosis. The infection can go as far as necrosis of the cartilage, which creates a loss of the framework, the main element of strictures; it can also produce arthritis, resulting in arytenoid immobility [3].

Laryngeal tuberculosis is diagnosed on clinical, radiological and bacteriological arguments (direct microscopic examinations, cultures, identification of the bacillus and antibiogram) and on characteristic anatomopathological signs (epithelio-giganto-cellular granuloma with caseous necrosis) [9]. Endoscopically, the budding appearance of tuberculosis is nonspecific and does not usually distinguish it macroscopically from squamous cell carcinoma [11, 12]. However, some aspects should suggest the diagnosis: presence of several lesions in islands, destructive nature, especially of the free edge of the epiglottis. Note that subglottic involvement is rare. Immobility of one or both vocal cords is common with involvement of the cricoarytenoid joint [13]. In case of suspicion of laryngeal tuberculosis, biopsies sent both in anatomo-pathology (search for epithelio-giganto-cellular granulomas with caseous necrosis) and in bacteriology with inoculation on Löwenstein-Jensen medium are the key to the diagnosis [14].

The reference imaging is cervical CT scan with injection of contrast product, in parenchymal windows and bone windows [15]. Even if the lesions observed are not specific, the diagnosis of laryngeal tuberculosis should be systematically evoked in the event of bilateral and diffuse lesions without destruction of the laryngeal architecture, even more so if a pulmonary localization is known or strongly suspected. The latter can generally be easily differentiated from a secondary location on conventional imaging (false negative chest $\mathrm{x}$-ray in less than $5 \%$ of cases) or better by $\mathrm{C} \mathrm{T}$ scan (often excavated opacities and / or mediastinal lymphadenopathy) [11]. The other signs suggesting tuberculosis rather than squamous cell carcinoma are amputation of the free edge of the epiglottis, a discrepancy between the importance of clinical lesions (budding) and radiological images (shallow infiltration), and finally the preservation of the hyo-thyro-epiglottic compartment (HTE) and paraglottic spaces [15]. Several classifications of strictures have been proposed. Classifications combining anatomical and functional data are the most useful in guiding therapeutic indications. The classification is made from morphological (clinical examination including indirect laryngoscopy, imaging, endoscopy) and dynamic data [3]. The most widely used classifications in the international literature use the classic terms glottic, subglottic, supraglottic stenosis. Various classifications incorporating the extent and severity of the lesions have been proposed for prognostic purposes [16, 17]. The management of laryngeal stenosis in adults is complex in its indications and in its implementation. It involves several diagnostic and therapeutic steps, involving several general anesthesias and, in some cases, requiring a tracheotomy as was in our case. Trans-oral laser reconstructive microsurgery (R-TLM) can effectively treat this type of stenosis and decannulate the patient [16-18]. Given the absence of this type of treatment in our country, symptomatic treatment such as a tracheotomy was carried out.

The total duration of the treatment before reaching decannulation is very often several months [2]. The patient and his entourage will be usefully informed of these binding and inevitable aspects. In this respect, a comprehensive description of the treatment plan is beneficial to them.

\section{Conclusion}

Laryngeal stenosis secondary to multifocal tuberculosis remains a rare and dreadful pathology. Clinically, it manifests itself by nonspecific signs, limited to the larynx or part of a 
multi-organ pathology. Treatment is often difficult, with several treatment options. The early and adequate management of this pathology conditions the therapeutic success.

\section{Declaration of Interests}

All the authors do not have any possible conflicts of interest.

\section{References}

[1] Madeleine M, Daniel B. Traité d'ORL, médecine - sciences Flammarion, 2008, (62) 461-472 p.

[2] Mercy G, Yves J, Christos I, Philippe M. Management of severe pediatric subglottic stenosis with glottic Involvement. J Thorac Cardiovasc Surg 2010; 139: 411-17.

[3] Lacau St Guily J, Périé S et Coiffier L. Sténoses laryngées de l'adulte. Encycl Méd Chir (Editions Scientifiques et Médicales Elsevier SAS, Paris, tous droits réservés), Otorhino-laryngologie, 20-735-A-10, 2003, 15 p.

[4] Tanya K, Meyer MD; Jeffrey Wolf, MD. Lysis of Interarytenoid Synechia (Type I Posterior Glottic Stenosis): Vocal Fold Mobility and Airway Results. The American Laryngological, Rhinological and Otological Society, Inc. 2011; 121: 2165-71.

[5] Auerbach O. Laryngeal tuberculosis. Arch Otolaryngol 1946; 44: 191-201.

[6] Dye C, Scheele S, Dolin P. Global burden of tuberculosis, estimated incidence, prevalence, and mortality by country. JAMA 1999; 282: 677-686.

[7] Millard FJ. The rising incidence of tuberculosis. $J$ R Soc Med 1996; 89: 497-500.
[8] Spence DP, Hotchkiss J, Williams CS, Davies PD. Tuberculosis and poverty. Br Med J 1993; 307: 759-761.

[9] Portier F, Cartry F et Nowak C. Tuberculose laryngée. Encycl Méd Chir (Editions Scientifiques et Médicales Elsevier SAS, Paris, tous droits réservés), Oto-rhino-laryngologie, 20-575-A10, 2003, 4 p.

[10] Ramandan HH, Tarazi AE, Baroudy FM. Laryngeal tuberculosis: presentation of 16 cases and review of the literature. J Otolaryngol 1993; 22: 39-41.

[11] Delap TG, Lavy JA, Alusi G, Quiney RE. Tuberculosis presenting as a laryngeal tumour. J Infect 1997; 34: 139-141.

[12] Lightfoot SA. Laryngeal tuberculosis masquerading as carcinoma. J Am Board Fam Pract 1997; 10: 374-376.

[13] Konishi K, Yamane H, Iguchi H, Nakagawa T, Shibata S, Takayama $M$ et al. Study of tuberculosis in the field of otorhinolaryngology in the past 10 years. Acta Otolaryngol [suppl] 1998; 538: 244-249.

[14] Richter B, Fradis M, Kohler G, Ridder GJ. Epiglottic tuberculosis: differential diagnosis and treatment. Case report and review of the literature. Ann Otol Rhinol Laryngol 2001; 110: 197-201.

[15] Moon WK, Han MH, Chang KH, Im JG, Kim HJ, Sung KJ et al. CT and MR imaging of head and neck tuberculosis. Radiographics 1997; 17: 391-402.

[16] Lano CF Jr, Duncavage JA, Reinisch L, Ossoff RH, Courey MS, Netterville JL. Laryngotracheal reconstruction in the adult: aten year experience. AnnOtol Rhinol Laryngol 1998; 107: 92-97.

[17] McCaffrey TV. Managementof laryngotracheal stenosis on the basis of site and severity. Otolaryngol Head Neck Surg 1993; 109 (3 Pt 1): 468-473.

[18] Pearson FG. Technique of management of subglottic stenosis. Chest Surg Clin N Am 1996; 6: 683-692. 